\title{
Transforaminal Lumbar Interbody Fusion with Unilateral Pedicle Screw and Contralateral Percutaneous Transfacet Screw Fixation for the Treatment of Lumbar Degenerative Disorders
}

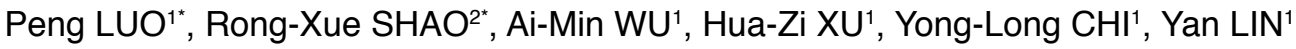 \\ ${ }^{1}$ Second Affiliated Hospital of Wenzhou Medical University, Department of Spinal Surgery, China \\ ${ }^{2}$ Zhejiang Chinese Medical University, Department of Spinal Surgery, China \\ *These authors contributed equally to this work and should be considered co-first authors.
}

\section{ABSTRACT}

AIM: To assess the feasibility and efficacy of TLIF with unilateral pedicle screw and contralateral percutaneous lumbar transfacet screw fixation for the treatment of lumbar degenerative disorders.

MATERIAL and METHODS: A series of computed tomography (CT) digital images from 60 patients (30 males and 30 females) with L3-S1 segments, were reconstructed in three dimensions using a software named Xelis 3D. Linear and angular measurements of the facets were recorded. Fifty-six patients were divided to either bilateral pedicle screw fixation (BPS) $(n=30)$ or unilateral pedicle screw and contralateral percutaneous transfacet screw fixation (UPFS) $(n=26)$ groups. The operating time, blood loss, length of hospital stay, clinical outcomes, fusion and complication rates were compared between the two groups.

RESULTS: The parameters of the facets we recorded were no significant differences between the left and right sides ( $p>0.05$ ). No statistically significant differences between males and females were observed, except the maximum width of superior facet at $L 4$ and S1, and length of screw at all levels. Intraoperative parameters, such as operating time, blood loss and postoperative hospital stay had significantly larger in the BPS group than UPFS group $(p<0.05)$. No significant differences were found between the two groups in clinical results, fusion and complication rates ( $p>0.05)$.

CONCLUSION: Because of the similar clinical outcomes but less operative time, blood loss and length of postoperatively hospital stay, unilateral pedicle screw and contralateral percutaneous lumbar transfacet screw fixation might be an attractive technique compared to bilateral pedicle screw fixation for treating lumbar degenerative disorders with TLIF. Nevertheless differences of the anatomical parameters for the lumbosacral facets fixation between Chinese and Westerners should be noted.

KEYWORDS: Lumbar facet anatomy, Percutaneous transfacet screw, Lumbar spine fusion, Minimally invasive

\section{INTRODUCTION}

$\mathrm{T}$ Transforaminal lumbar interbody fusion (TLIF) is regaining popularity as a method for the treatment of a variety of lumbar disorders $(9,12,14)$, including lumbar disc herniation, spinal stenosis, segmental instability and degenerative spondylolisthesis. One of the disadvantages of traditional lumbar fusion, however, is the extensive soft tissue dissection that may lead to increase postoperative pain, lengthen recovery time, impair spinal function $(3,7)$. In 2005, Jang and Lee (6) proposed a technique of TLIF, involving ipsilateral pedicle screw and contralateral translaminar screw 



Figure 1: The axial scan of $L 4$. A: the maximum width of the superior facet; $\alpha$ : the angle of the superior facet relative to midline. B: the maximum width of the inferior facet.

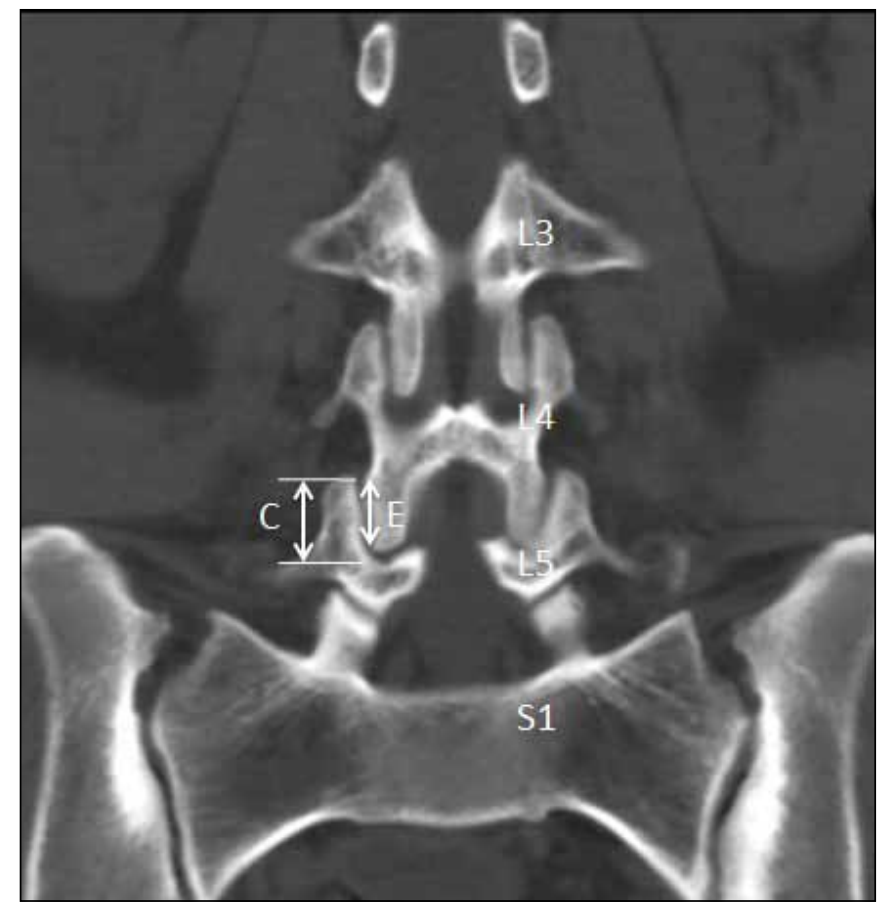

Figure 2: The coronal scan of lumbosacral vertebrae (C: the height of superior facet; $\mathrm{E}$ : the height of inferior facet).

fixation, which was proven to cause less estimated blood loss and soft tissue injury than conventional TLIF. With the development of minimally invasive procedures, Hsiang (4) recently presented a novel management of unilateral pedicle screw fixation combined with contralateral percutaneous transpedicular facet screw (UPFS) construct that reduced the amount of normal tissue injury further. Nevertheless, there is still insufficient clinical data on this new method globally, especially in the Chinese population. The purposes of this study are not only to demonstrate the feasibility of this surgery by investigating the morphometry of the lumbosacral facets of Chinese adults, but also to compare the relevant parameters between bilateral pedicle screw fixation (BPS) and unilateral pedicle screw - contralateral percutaneous transfacet screw fixation for the treatment of lumbar degenerative disorders.

\section{MATERIAL and METHODS}

\section{Imaging Materials and Measurement}

The 3D computed tomography (CT) images, including the healthy L3-S1 segments of 60 consecutive adult patients with an equal distribution of male and female, and whose age ranged from 19 to 52 years (average $31.23 \pm 6.88$ and $30.43 \pm 7.33$ years old, respectively, $p>0.05$ ), were obtained from the database of our hospital. Patients with ankylosing spondylitis, scoliosis, or pars defects were excluded from the study. All patients were scanned using a CT scanner (Brilliance 16; Philips, Inc. USA). The scans contained a slice and spacing thickness of $1 \mathrm{~mm}$ each. The data were reconstructed by a software named Xelis 3D (Infinitt, Inc. South Korea). The following facet parameters were measured on the axial planes: the maximum width of superior facet $(A)$ and inferior facet $(B)$, angle of the superior facet relative to midline $(\alpha)$ (Figure 1). On the coronal planes, the height of superior facet $(C)$ and inferior facet $(E)$ were measured respectively (Figure 2). Then the starting point was defined as the intersection of a vertical line drawn at the medial aspect of the pedicles with the inferior endplate of the superior vertebra being fused on the AP view $(16,17)$ (Figure $3 A)$. The 3D CT imaging planes were concurrently manipulated which allowed the starting point to be observed on the sagittal, transverse and coronal planes at the same time (Figure 3B$D)$. The starting point was also observed on the lateral view of the 3D CT imaging, the multiplanar reconstruction mode was used to visually determine the trajectory of the transfacet screw from the starting point to the midpoint of the transfacet (Figure 4). On this reconstructed axial plane, the exit point was defined as the intersection of the line drawn across the starting point to midpoint of the superior facet to the lateral aspect of the pedicle. The distance from the entry point to exit point (the trajectory length $(E)$ ) was then measured, and the lateral angulation of the transfacet screw ( $\beta$ ) relative to the spinous process was measured on the same scan as well (Figure 5). The caudal angulation of the transfacet screw $(\gamma)$ relative to a line parallel to the inferior endplate of the corresponding superior vertebra, was measured on the lateral view of the lumbosacral vertebrae (Figure 4). Those procedures were repeated for each patient's CT scan at the L3, L4, L5 and S1 levels bilaterally. 




Figure 3: Three-dimensional CT concurrent views in all planes manipulated to locate the starting point. A) the starting point was located at the intersection of a vertical line drawn at the medial aspect of the pedicles with the inferior endplate of the superior vertebra on the AP view. B-D) the sagittal, axial and coronal plane showing the position of the starting point respectively.

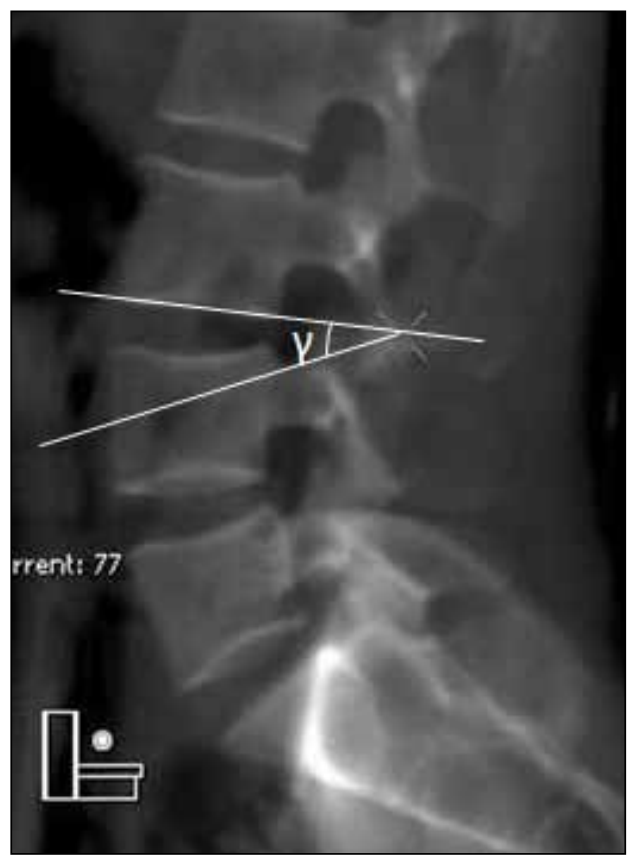

Figure 4: The lateral view of lumbosacral vertebrae. $\gamma$ : the caudal angulation of the transfacet screw relative to a line parallel to the inferior endplate of the corresponding superior vertebra.

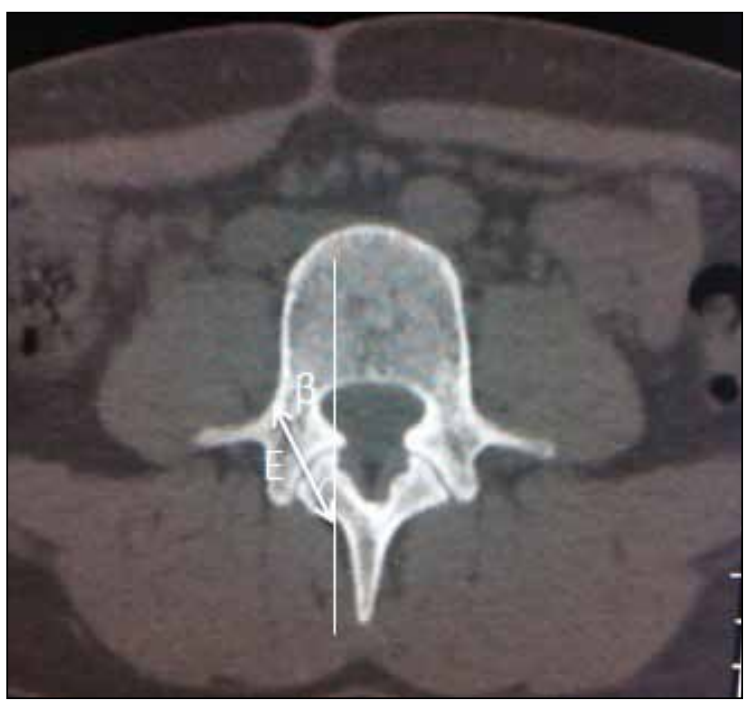

Figure 5: The reformed axial plane, from the starting point to the midpoint of the transfacet in the lateral view of lumbosacral vertebrae, indicating the trajectory of the transfacet screw (E: The trajectory length measured from entry point to exit point). 
Each measurement was taken three times and a mean value was calculated.

\section{Clinical Materials and Surgical Technique}

A retrospective review was conducted for 56 consecutive patients who underwent TLIF with unilateral pedicle screw and contralateral facet screw fixation (26 patients from November 2009 to July 2010) or bilateral pedicle screw fixation (30 patients from August 2010 to April 2011) at a single institution. All the operations were done by a single surgeon. All patients underwent at least 6 months of nonoperative management before surgery. Patients with previous spinal instrumentation, multilevel pathologies, spinal tumor, spinal infections, acute spinal trauma or fractures, and higher grades (> Grade-l) of spondylolisthesis were excluded. The patients' demographic characteristics were listed in Table I. The minimum duration of follow-up required was 18 months.

\section{Unilateral TLIF Approach}

Under general anesthesia, patients were placed in the prone position with an empty abdomen. The approach was carried out unilateral on the symptomatic side. A midline incision, about 6-7 cm, was performed at the level of interest with radiological confirmation by $\mathrm{C}$-arm fluoroscopy. The posterior spinal elements of the operation side, including facet joint and lamina were exposed, extending one vertebrae above and below the involved segments. Pedicle screws were implanted into the vertebral body, and the inferior and superior articular processes and part of the lamina were removed by using an osteotome and power-drill. The ligamentum flavum was removed to expose the lateral border of the ipsilateral nerve root. With regard to the 5 patients who had bilateral radiculopathy, the central canal and even contralateral lateral recess could be decompressed (10). After complete decompression of the neural structures, discectomy and end plate decortication were performed followed by transforaminal insertion of an intervertebral tantalum mesh cage packed with autograft materials within the disc space. The compression was achieved across the pedicle screws and rod with set screws, and optimal hardware placement was confirmed by intraoperative fluoroscopy.

\section{Percutaneous Facet Screw Placement}

A $1 \mathrm{~cm}$ parasagittal incision was made at about one to two levels cephalad to the level of interest. In the A/P view of fluoroscopy, the starting point was located at the intersection of a vertical line drawn at the medial aspect of the pedicles with the inferior endplate of the superior vertebra. AK-wire was implanted percutaneously into the starting point. The lateral view indicated that the ideal trajectory aiming for the center of the contralateral superior articular process of inferior vertebra in order to reach the lateral aspect of pedicle. The lateral and caudal angulation of the transfacet screw was based on our radiological study. A protective tube was implanted along the K-wire direction. Then, the K-wire was inserted along the expected trajectory to the pedicle of inferior vertebrae with a power-drill. Finally, an appropriate length of cannulated screw was placed across the K-wire through the protective tube. The wounds were closed in the standard manner (Figure 6).

Table I: Demographic Characteristics

\begin{tabular}{|c|c|c|c|}
\hline & BPS & UPFS & $\mathbf{p}$ \\
\hline Female & $9(30.0 \%)$ & $11(42.3 \%)$ & 0.34 \\
\hline Average age (years) & $54.80 \pm 10.23$ & $49.77 \pm 9.52$ & 0.06 \\
\hline Diagnosis for operation & & & 0.57 \\
\hline Foraminal stenosis & $10(33.3 \%)$ & $8(30.8 \%)$ & \\
\hline Lumbar disc herniation & $9(30.0 \%)$ & $5(19.2 \%)$ & \\
\hline Recurrent disc herniation & $5(16.7 \%)$ & $3(11.5 \%)$ & \\
\hline Degenerative disc disease & $1(3.3 \%)$ & $3(11.5 \%)$ & \\
\hline$\llcorner 4-5$ & $12(40.0 \%)$ & $13(50.0 \%)$ & \\
\hline L5-S1 & $8(26.7 \%)$ & 10 (38.5\%) & \\
\hline Bilateral radiculopathy & $8(26.7 \%)$ & $5(19.2 \%)$ & 0.51 \\
\hline Follow-up (months) & $30.50 \pm 7.75$ & $31.31 \pm 8.40$ & 0.71 \\
\hline
\end{tabular}

BPS: Bilateral pedicle screw, UPF: Unilateral pedicle and contralateral percutaneous facet screw. 


\section{Bilateral TLIF Approach}

A standard procedure was used involving a midline incision and bilateral pedicle screw fixation. Unilateral facetectomy and decompression was performed on the symptomatic side, and the opposite facet joint remained intact. For those patients who had bilateral radiculopathy, facetectomy and decompression of both sides were carried out.

\section{Data Collection}

The operative time, blood loss, and length of hospitalization were recorded for each patient. Plain radiographs and CT scans were performed at last follow-up for each patient to evaluate the proper screw location and the fusion rate (Figure 7). Fusion rates were assessed with the Bridwell grading system which was composed of the following categories and grades: fused with remodeling and trabeculae present (Grade I); graft intact, not fully remodeled and incorporated, but no lucency present (Grade II); graft intact, potential lucency present at top and bottom of graft (Grade III); and fusion absent with collapse/ resorption of the graft (Grade IV) (2). Clinical outcomes were collected preoperatively and at last follow-up using the Visual analogue scale (VAS) pain scale for the back and leg and the Oswestry disability index scores (ODI). We certify that all applicable institutional and governmental regulations concerning the ethical use of human volunteers were followed during the course of this research.

\section{Statistical Analysis}

Statistical analysis, including mean values and standard deviations, were carried out using SPSS software v17.0 (SPSS Inc. Chicago, Illinois). Independent-samples t-tests were used for comparing the radiological parameters between males and females, and the continuous variables between groups, such as operating time, blood loss, length of hospital stay, VAS scores, and ODI. Paired-samples t-tests were performed within each group to compare the results before and after surgery, and these were also used to compare the radiological parameters between the left and right side. Chi-square tests were used for categorical variables such as gender, diagnosis, operative level and fusion status. Differences were considered statistically significant at $p<0.05$.

\section{RESULTS}

There were no significant differences between the measured values on the left and right sides $(p>0.05)$. Thus the mean corresponding values for all levels were accounted and presented in Table II and III. There were no statistically significant differences between males and females, except the maximum width of the superior facet at L4 and S1, and trajectory length at all levels. The trajectory lengths were $33.47 \pm 3.21 \mathrm{~mm} ; 37.43 \pm 4.53 \mathrm{~mm} ; 44.67 \pm 4.06 \mathrm{~mm}$ for males and $28.24 \pm 3.68 \mathrm{~mm} ; 33.24 \pm 5.32 \mathrm{~mm} ; 42.20 \pm 4.74 \mathrm{~mm}$ for females at the L3-4, L4-5 and L5-S1 respectively $(p<0.05)$.
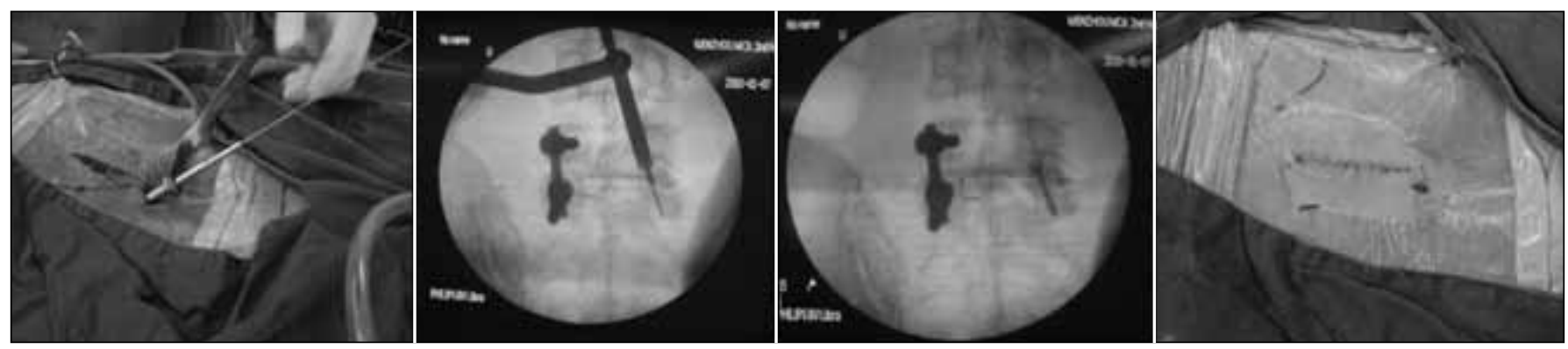

Figure 6: The procedure of percutaneous facet screw implantation.


Figure 7: Postoperative (6 months) x-ray and CT scans. 
Mean length of follow-up for the BPS and UPFS groups were $30.50 \pm 7.75$ months (range 18 to 46 months) and $31.31 \pm 8.40$ months (range from 18 to 48 months), respectively. There were no significant differences between the two groups in terms of patient demographics (Table I). With respect to intraoperative parameters, patients in the BPS group had significantly longer operating time, more blood loss and postoperative hospital stay $(p<0.05)$ (Table IV). No significant differences of visual analog scale for back and leg pain and Oswestry Disability Index scores were observed between two groups ( $p>0.05$ ), although all of those records had improved postoperatively in each group $(p<0.05)$ (Table IV).

Until time of the last follow-up, fusion grades in the BPS group were Grade I in $70.0 \%(n=21)$, Grade II in $26.7 \%(n=8)$, Grade III in 3.3\% ( $n=1)$, and Grade IV in $0 \%$ of patients; in the UPFS group, fusion grades were Grade I in $88.5 \%(n=23)$, Grade II in $11.5 \%(n=3)$, Grade III and Grade in IV in $0 \%$ of patients, respectively. Radiological evidence showed that 29 (96.7\%) patients achieved successful fusion in the BPS group, and 26 (100\%) patients in the UPFS group, showing no statistically significant difference $(p>0.05)$.
No serious complication was found in the BPS or UPFS group. There were 2 cases of postoperative superficial wound infection in the BPS group. Infections were completely controlled by using antibiotics and daily dressing. One case of cerebrospinal fluid leak developed in the BPS group. Neither group showed any signs of screw failure.

\section{DISCUSSION}

Transforaminal lumbar interbody fusion (TLIF), as an operative technique with all the advantages of anterior lumbar interbody fusion (ALIF) and posterior lumbar interbody fusion (PLIF) methods and a much lower complication rate, has been applied widely in spinal surgery $(5,13)$. Slucky et al. (15) demonstrated that TLIF with unilateral pedicle screw fixation only provided half of total strength of bilateral pedicle screw fixation by using human cadaveric specimens, but unilateral fixation with contralateral translaminar screw provided a biomechanical stability comparable with bilateral pedicle screw fixation. Su et al. (16) presented an anatomic study of the lumbar facet and described an ideal starting point and trajectory for percutaneous transfacet fixation. Based on

Table II: Measurements of Facet Anatomy

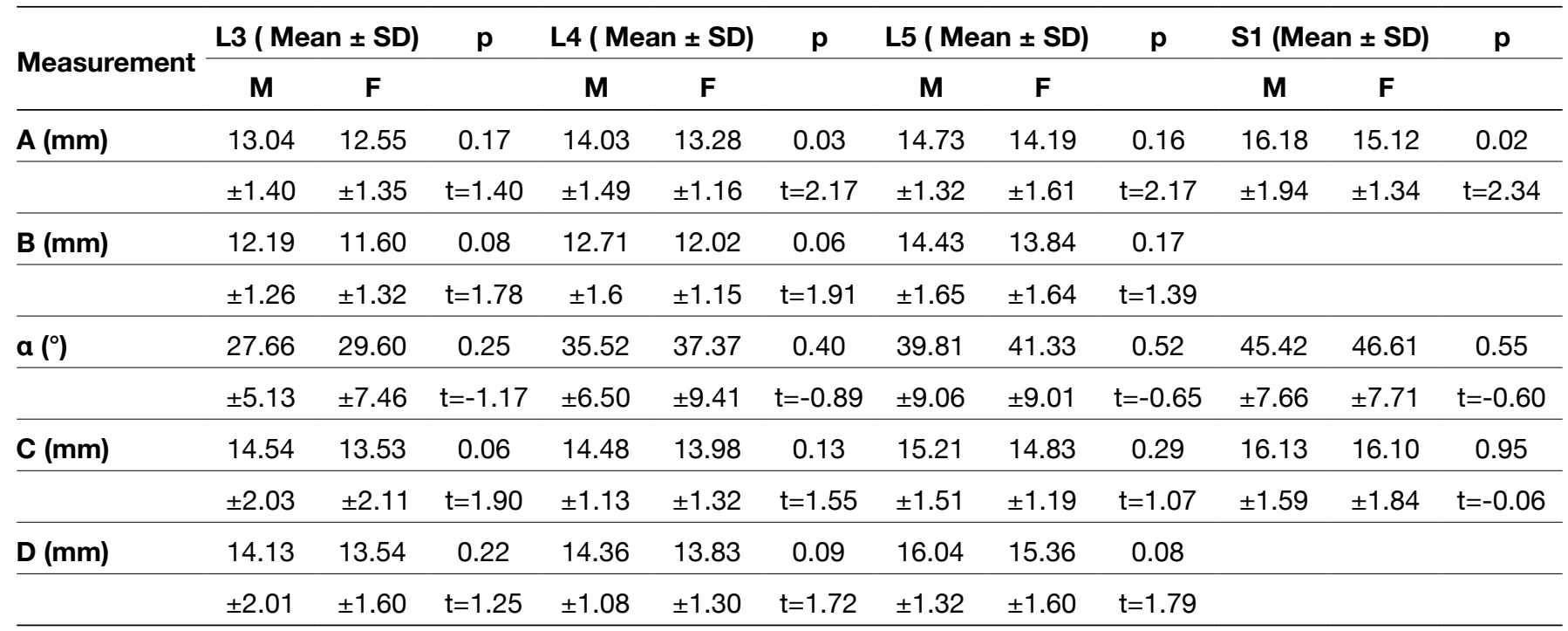

Table III: Measurements of L3-S1 Segment

\begin{tabular}{|c|c|c|c|c|c|c|c|c|c|}
\hline Measurement & \multicolumn{2}{|c|}{ L3-4 (Mean \pm SD) } & $\mathbf{p}$ & \multicolumn{2}{|c|}{ L4-5 (Mean \pm SD) } & $\mathbf{p}$ & \multicolumn{2}{|c|}{ L5-S1 (Mean \pm SD) } & $\mathbf{p}$ \\
\hline $\mathrm{E}(\mathrm{mm})$ & \pm 3.21 & \pm 3.68 & $t=5.87$ & \pm 4.53 & \pm 5.32 & $t=3.28$ & \pm 4.06 & \pm 4.74 & $t=2.17$ \\
\hline $\boldsymbol{\beta}\left({ }^{\circ}\right)$ & 18.43 & 20.09 & 0.18 & 19.97 & 20.79 & 0.43 & 21.17 & 22.03 & 0.25 \\
\hline \multirow[t]{2}{*}{$\underline{\gamma\left({ }^{\circ}\right)}$} & 37.25 & 38.47 & 0.55 & 40.18 & 41.36 & 0.33 & 41.12 & 42.13 & 0.62 \\
\hline & \pm 6.11 & \pm 9.21 & $t=-0.60$ & \pm 4.16 & \pm 5.12 & $t=-0.91$ & \pm 8.03 & \pm 7.52 & $t=-0.53$ \\
\hline
\end{tabular}


this biomechanical data, Hsiang et al. (4) recently reported a novel technique of unilateral pedicle screw fixation combined with contralateral percutaneous transpedicular facet screw construction. They stated that the report served as a pilot study of this technique based on 40 patients but data on clinical outcomes such as disability scores and fusion rate were still inadequate. In order to demonstrate the feasibility of the percutaneous transpedicular facet screw fixation applied in Chinese adults, the morphometry of the lumbosacral facets of Chinese were measured.

Some of the measurements in our study had been performed by Su et al. (16) and the data of males and females were measured in our study, respectively. According to the parameters we measured, there were no significant differences between males and females, besides the maximum width of the superior facet at $L 4$ and S1, maximum depth of the superior facet at L5, and the trajectory length at all levels. The maximum width of the superior and inferior facet, and the height of the superior facet and inferior facet in our study, were 1-2 $\mathrm{mm}$ smaller than those of Su et al. (16) at the corresponding vertebral body. The maximum width of the superior and inferior facet joints and the maximum height of the superior and inferior facet joints can provide an adequate surface area for placement of a screw across the joint. The L2-3 segment was not analyzed in the present study because it was impossible to place an ipsilateral pin through the center of the facet with the vertical orientation of the facet joint (16). The transverse facet angles $(\alpha)$ we measured as $27.66 \pm 5.13^{\circ}$ (male) at L3 and $35.52 \pm 6.50^{\circ}$ (male) at L4 were almost $5^{\circ}$ larger than those of Su who reported $21.3 \pm 10.0^{\circ}$ at $L 3$ and $29.9 \pm 10.5^{\circ}$ at $L 4$, while the angles we measured at $\mathrm{L} 5$ and $\mathrm{S} 1$ were similar to those Su et al. reported as $38.0 \pm 9.6^{\circ}$ at L5 and $45.8 \pm 9.8^{\circ}(16)$. We found that the transfacet screw in the axial plane should be laterally angulated $(\beta) 18.43 \pm 4.32^{\circ}$ (male) at L3-4, $19.97 \pm 3.71^{\circ}$ (male) at L4-5, and $21.17 \pm 2.41^{\circ}$ (male) at L5-S1, and in the sagittal plane it should be caudally angulated $(\gamma) 37.25 \pm 6.11^{\circ}$ (male) at L3-4, 40.18 $\pm 4.16^{\circ}$ (male) at L4-5, and $41.12 \pm 8.03^{\circ}$ (male) at L5-S1, which are $4^{\circ}$ and $10^{\circ}$ larger than Su's measurements at the corresponding vertebral body, respectively. There are therefore many differences in anatomical parameters of the lumbosacral facet fixation between Chinese and Westerners.

Based on our anatomical research, 26 patients who suffered from lumbar degenerative disorders underwent transforaminal lumbar interbody fusion with a combination of unilateral pedicle screw and contralateral percutaneous pedicle screw fixation. The average surgical time, blood loss, and length of hospitalization was $132.81 \pm 20.80$ minutes, $267.31 \pm 80.43 \mathrm{ml}$ and $6.15 \pm 1.93$ days, larger than those of Hsiang reported as 124 minutes, $140 \mathrm{ml}$ and 3 days (4). These differences may explain the reason we used the traditional open TLIF approach in our patients while in Hsiang's research, they used the Wiltse approach, which is a minimally invasive surgery leading between the multifidus and longissimus muscle groups to the facet joint. The improvement of the VAS pain scale for the back and leg and the Oswestry disability index score revealed a good clinical outcome of our patients. The percutaneous placement of transfacet screws has several advantages, minimizing injury of paraspinal musculature, making blood

Table IV: Comparison of Outcome Measurements Between Two Groups

\begin{tabular}{|c|c|c|c|}
\hline & BPS $(n=32)$ & UPFS (n=26) & $\mathbf{p}$ \\
\hline Operative time (minutes) & $198.00 \pm 25.65$ & $132.81 \pm 20.80$ & $0.004^{*}$ \\
\hline Hospital stay (days) & $8.13 \pm 3.76$ & $6.15 \pm 1.93$ & $0.02^{*}$ \\
\hline Blood loss (mL) & $463.67 \pm 98.59$ & $267.31 \pm 80.43$ & $0.00^{\star}$ \\
\hline \multicolumn{4}{|l|}{ VAS for back pain } \\
\hline Preop & $7.07 \pm 1.26$ & $6.84 \pm 1.38$ & 0.53 \\
\hline Postop & $2.50 \pm 0.82$ & $2.77 \pm 0.99$ & 0.27 \\
\hline \multicolumn{4}{|l|}{ VAS for leg pain } \\
\hline Preop & $6.73 \pm 1.11$ & $7.04 \pm 1.46$ & 0.38 \\
\hline Postop & $2.33 \pm 1.09$ & $2.54 \pm 0.95$ & 0.46 \\
\hline \multicolumn{4}{|l|}{ ODI } \\
\hline Preop & $41.60 \pm 9.13$ & $38.19 \pm 7.09$ & 0.13 \\
\hline Postop & $13.40 \pm 5.20$ & $11.23 \pm 4.60$ & 0.11 \\
\hline Fusion & $29(96.7 \%)$ & $26(100 \%)$ & 0.34 \\
\hline Complications & $3(10.0 \%)$ & $0(0 \%)$ & 0.09 \\
\hline
\end{tabular}

VAS: Visual analog scale, ODI: Oswestry disability index, BPS: Bilateral pedicle screw, UPFS: Unilateral pedicle and contralateral percutaneous facet screw. Significant differences were found $(p<0.05)$ before and after operation for VAS, ODI in both groups.

*Indicates significant statistical difference between groups. 
loss negligible, and mitigating postoperative pain, for instance. The trajectory of the instrument reduces the potential for canal violation and nerve injury. The lag screw may compress the facet joint, theoretically increasing segmental stiffness and the potential for facet arthrodesis. Su et al. (16) recommends ipsilateral transfacet fixation as a viable alternative method for the L3-4 level and below. It is worth noting, according to our clinical experiences, that it was a little difficult for us to implant the screw right through the center of the facet from the ipsilateral side at the L3-4 level because of the small angle of the facet joint and the obstacle of the spinous process, but it did not seem to impact the effectiveness of the fixation.

Our results suggest that the technique (UPFS) we used in the study will likely prove an attractive method compared to traditional bilateral pedicle screw fixation. No significant differences were found between the two groups in terms of clinical outcomes but the operative time, blood loss and length of postoperative hospital stay in rgw UPFS group was less than in the BPS group. Some may doubt that whether a unilateral approach can allow bilateral decompression. In fact, by compressing the thecal sac cautiously, contralateral decompression can be performed through a unilateral approach by using the rongeur (10). Five of our patients who had bilateral radiculopathy showed a satisfactory clinical release for both back and leg pain after bilateral decompression using a unilateral approach. Nevertheless, there are some disadvantages of our technique. Compared with Hsiang's method (4), the open approach for lumbar fusion will inevitably cause an injury to the mid-line structures, such as ligaments and paraspinal muscles, which may cause postoperative dysfunction of the spinal muscles, leading to prolonged postoperative back pain $(1,8,11)$. Patients with more than grade 1 spondylolisthesis, spondylolysis, laminal fractures, severe osteoporosis and facet arthropathy, and severe lumbar spinal stenosis requiring extensive bilateral decompression probably are not good candidates for this management. Further analysis and follow-up will be required to determine the long-term outcomes.

\section{- CONCLUSION}

Transforaminal lumbar interbody fusion for treating lumbar degenerative disorders with unilateral pedicle screw and contralateral percutaneous lumbar transfacet screw fixation might be an attractive technique compared to bilateral pedicle screw fixation because of the similar clinical outcomes but less operative time, blood loss and length of postoperative hospital stay. Nevertheless there were many differences in the anatomical parameters for lumbosacral facet fixation between Chinese and Westerners.

\section{- REFERENCES}

1. Arts MP, Nieborg A, Brand R, Peul WC: Serum creatine phosphokinase as an indicator of muscle injury after various spinal and nonspinal surgical procedures. J Neurosurg Spine 7:282-286, 2007
2. Bridwell KH, Lenke LG, McEnery KW, Baldus C, Blanke K: Anterior fresh frozen structural allografts in the thoracic and lumbar spine. Do they work if combined with posterior fusion and instrumentation in adult patients with kyphosis or anterior column defects? Spine (Phila Pa 1976) 20:1410-1418, 1995

3. Gejo R, Matsui $H$, Kawaguchi $Y$, Ishihara $H$, Tsuji $H$ : Serial changes in trunk muscle performance after posterior lumbar surgery. Spine (Phila Pa 1976) 24:1023-1028, 1999

4. Hsiang J, Yu K, He Y: Minimally invasive one-level lumbar decompression and fusion surgery with posterior instrumentation using a combination of pedicle screw fixation and transpedicular facet screw construct. Surg Neurol Int 4:125, 2013

5. Humphreys SC, Hodges SD, Patwardhan AG, Eck JC, Murphy $\mathrm{RB}$, Covington LA: Comparison of posterior and transforaminal approaches to lumbar interbody fusion. Spine (Phila Pa 1976) 26:567-571, 2001

6. Jang JS, Lee SH: Minimally invasive transforaminal lumbar interbody fusion with ipsilateral pedicle screw and contralateral facet screw fixation. J Neurosurg Spine 3:218-223, 2005

7. Kawaguchi $Y$, Matsui $H$, Tsuji H: Back muscle injury after posterior lumbar spine surgery. A histologic and enzymatic analysis. Spine (Phila Pa 1976) 21:941-944, 1996

8. Kawaguchi Y, Yabuki S, Styf J, Olmarker K, Rydevik B, Matsui $\mathrm{H}$, Tsuji H: Back muscle injury after posterior lumbar spine surgery. Topographic evaluation of intramuscular pressure and blood flow in the porcine back muscle during surgery. Spine (Phila Pa 1976) 21:2683-2688, 1996

9. Lowe TG, Tahernia AD: Unilateral transforaminal posterior lumbar interbody fusion. Clin Orthop Relat Res 394:64-72, 2002

10. Mao L, Zhao J, Dai KR, Hua L, Sun XJ: Bilateral decompression using a unilateral pedicle construct for lumbar stenosis. Int Orthop 38:573-578, 2014

11. Min SH, Kim MH, Seo JB, Lee JY, Lee DH: The quantitative analysis of back muscle degeneration after posterior lumbar fusion: Comparison of minimally invasive and conventional open surgery. Asian Spine J 3:89-95, 2009

12. Moskowitz A: Transforaminal lumbar interbody fusion. Orthop Clin North Am 33:359-366, 2002

13. Rapan S, Jovanovic S, Gulan G: Transforaminal lumbar interbody fusion (TLIF) and unilateral transpedicular fixation. Coll Antropol 34:531-534, 2010

14. Rosenberg WS, Mummaneni PV: Transforaminal lumbar interbody fusion: Technique, complications, and early results. Neurosurgery 48:569-575, 2001

15. Slucky AV, Brodke DS, Bachus KN, Droge JA, Braun JT: Less invasive posterior fixation method following transforaminal lumbar interbody fusion: A biomechanical analysis. Spine $J$ 6:78-85, 2006

16. Su BW, Cha TD, Kim PD, Lee J, April EW, Weidenbaum M, Albert TJ, Vaccaro AR: An anatomic and radiographic study of lumbar facets relevant to percutaneous transfacet fixation. Spine (Phila Pa 1976) 34:E384-390, 2009

17. Voyadzis JM, Anaizi AN: Minimally invasive lumbar transfacet screw fixation in the lateral decubitus position after extreme lateral interbody fusion: $A$ technique and feasibility study. $J$ Spinal Disord Tech 26:98-106, 2013 